Ausschreibung

\section{Förderpreis für Krebsforschung}

Der Vorstand des Deutschen Krebsforschungszentrums (DKFZ) schreibt in diesem Jahr wieder bundesweit den mit $10000 €$ dotierten Förderpreis der Walther und Christine Richtzenhain-Stiftung aus. Mit dem Preis sollen richtungsweisende wissenschaftliche Arbeiten auf dem Gebiet der translationalen Krebsforschung ausgezeichnet werden. Im Auswahlverfahren werden wissenschaftliche Arbeiten aus deutschen Forschungsinstituten berücksichtigt, die in den Jahren 2014 und 2015 veröffentlicht oder zur Veröffentlichung angenommen wurden. Der Schwerpunkt soll auf dem erfolgreichen Transfer von Forschungsergebnissen in mögliche klinische Anwendungen liegen. Es können mehrere inhaltlich zusammengehörige Arbeiten eingereicht werden. Bei Veröffentlichungen mit mehreren Autoren wird darum gebeten, die Anteile der Kandidaten an der Publikation darzulegen.

Bevorzugt werden Bewerbungen von jungen Nachwuchswissenschaftlern. Der Abschluss der Promotion sollte nicht länger als 10 Jahre zurückliegen. Bewerbungen oder Vorschläge sollen neben einem Exemplar der Arbeit einen knappen Lebenslauf des Wissenschaftlers sowie das Publikationsverzeichnis der letzten 5 Jahre beinhalten. Die Auszeichnung wird jährlich abwechselnd an Doktoranden in Heidelberger Forschungsstätten und an Wissenschaftler aus der gesamten Bundesrepublik Deutschland vergeben. Die Auswahl der preiswürdigsten Arbeit erfolgt durch ein Gremium von Fachgutachtern.

Die Bewerbungen oder Kandidatenvorschläge sind in 5-facher Ausführung auf CD-ROM bis zum 15. Juli 2016 einzureichen. Auf jeder CD sollte das Bewerbungsschreiben, ein Lebenslauf, eine Publikationsliste sowie die Arbeit selbst abgespeichert sein. Die Unterlagen sind zu adressieren an den wissenschaftlicher Vorstand des DKFZ:

Prof. Michael Boutros

Postfach 101949

69009 Heidelberg.

Nach einer Mitteilung des DKFZ, Heidelberg

\title{
Ist die Exazerbationsdauer ein Risikoparameter für Folgekomplikationen?
}

\author{
Bei exazerbierter COPD mit verlängerter Symptomdauer \\ oder deutlich verzögerter Erholung in der Spirometrie \\ muss von einer prinzipiell erhöhten Exazerbationsfrequenz \\ ausgegangen werden. Dies gilt besonders, wenn eine virale \\ Genese der initialen Verschlechterung wahrscheinlich ist, \\ wie G. C. Donaldson et al. anhand einer prospektiven Studie \\ aus England gezeigt haben. \\ Am J Respir Crit Care Med 2015; 192: 943-950
}

Bisher ist unklar, welche Aussagen die Dauer einer COPD-Exazerbation über den weiteren langfristigen Verlauf der Erkrankung zulässt. Verlängerte Exazerbationsphasen, eventuell auch nur spirometrisch nachweisbar, könnten eine zukünftig gesteigerte Exazerbationsgefahr indizieren. Dies wäre von hoher klinischer Relevanz, da dann ein erhöhter Überwachungsbedarf besteht. Es wird außerdem vermutet, dass das zukünftige Risiko einer Exazerbation erhöht ist, wenn sich der Patient als anfällig für Virusinfekte erweist.

\section{Aussagen zur Prognose \\ $\nabla$}

Die Autoren nutzten für ihre Analyse die Daten einer Londoner COPD-Kohorte. Diese enthält die klinischen und spirometrischen Angaben von 384 COPD-Patienten nach einer akuten Exazerbation über den Zeitraum von 1995 bis 2013. Die Betroffenen führten ein Symptomtagebuch. Eine Heimspirometrie ermöglichte die tägliche Erfassung des Peak Expiratory Flow (PEF)Werts. Die Forscher wollten klären, ob eine verlängerte Symptomdauer bzw. die Normalisierungszeit des PEFs Aussagen zur Prognose zulassen. Eine Virusgenese der initialen Verschlechterung verifizierten die Autoren anhand typischer klinischer Symptome. Auch die Auswirkung der Erkrankung auf die Lebensqualität der Betroffenen, gemessen mit dem St. George Respiratory Questionnaire (SGRQ) war Teil des Monitorings.

Insgesamt kam es bei 351 Patienten zu 3498 Exazerbationsepisoden. Im Mittel waren die Betroffenen nach 14,7 Tagen klinisch wieder symptomfrei. Verlängerte
Exazerbationsphasen stellten einen statistisch signifikanten Indikator für eine verkürzte exazerbationsfreie Folgezeit dar (Hazard Ratio: 1,004; p=0,013). Bei 257 Exazerbationen (7,3\%) normalisierten sich die PEF-Werte trotz Abklingen der Symptome erst im Langzeitverlauf (>99 Tage). Bei diesen Patienten reduzierte sich die $\mathrm{FEV}_{1}$ jährlich um $-33,6 \mathrm{ml}$ (typische COPD-Rate: $-22,8 \mathrm{ml} / \mathrm{Jahr}$ ). Die Konstellation war mit vermehrten virustypischen Symptomen assoziiert. Die verminderte Lebensqualität (SGRQ-Score) korrelierte mit der Zeitdauer der Exazerbation (0,2 Scoreeinheiten pro Tag; $\mathrm{p}=0,040$ ).

\section{Fazit}

Nach Angaben der Autoren sind COPDPatienten mit verlängerter Exazerbationssymptomatik risikoexponiert. Ihnen droht nicht nur ein schneller Rückfall, sie zeichnen sich auch durch eine erheblich verlängerte Kompromittierung ihrer respiratorischen Funktion aus, die ohne klinisches Korrelat fortbesteht. Als Konsequenz hieraus empfehlen die Autoren ein regelmäßiges engmaschiges PEF-Screening, besonders nach offenbar virusgetriggerten Exazerbationen.

\section{Dr. Horst Gross, Berlin}

